\title{
Música y filosofía (de la música): estado de la cuestión. Una revisión de problemas, discusiones y perspectivas ${ }^{1}$
}

Recepción: septiembre 2020. Aceptación: diciembre 2020.

\section{Resumen}

Este trabajo indaga los principales problemas, discusiones y perspectivas en que se configura actualmente la cuestión de la filosofía de la música. Se parte de una revisión de artículos publicados en revistas de alto impacto en el panorama internacional, recuperados a través de las bases de datos Scopus y Web of Science. Los análisis se complementan, eventualmente y según el criterio de relevancia, con otras publicaciones. Los resultados sugieren un campo en expansión y especialización que atraviesa la transición entre lo que podríamos denominar la perspectiva clásica de abordaje del objeto musical y las nuevas perspectivas, donde las fronteras son cada vez más frágiles entre filosofía de la música y estética de la música, musicología y abordajes de las ciencias sociales. Como planteamiento fundamental, se defiende que los temas y preocupaciones que aparecen en la literatura son actualizaciones de viejos problemas, antes usualmente asumidos desde la racionalidad formalista, de mayor tradición que las asunciones más fenomenológicas y hermenéuticas.

Palabras clave: filosofía de la música, formalismo, significado musical, música culta

\footnotetext{
${ }^{1}$ Este trabajo se desprende de la tesis doctoral "Música líquida: mediaciones de la canción contemporánea en el mundo de la vida", que presenta Arango, con la dirección de Roncallo-Dow, en el doctorado en filosofía de la Universidad Pontificia Bolivariana, Medellín, Colombia. El proyecto es cofinanciado por el Sistema de Investigación, Desarrollo e Innovación-SIDi- de la Universidad Católica de Oriente, Antioquia, Colombia.
} 


\title{
Música e filosofia (da música): estado da questão. Uma revisão de problemas, discussões e perspetivas
}

\section{Resumo}

Este trabalho indaga os principais problemas, discussões e perspetivas em que se configura atualmente a questão da filosofia da música. Baseia-se numa revisão de artigos publicados em revistas de alto impacto no panorama internacional, recuperados através das bases de dados Scopus e Web of Science. As análises complementam-se, eventualmente e segundo o critério de relevância, com outras publicações. Os resultados sugerem um campo em expansão e especialização que atravessa a transição entre o que poderíamos chamar a perspetiva clássica de aproximação ao objeto musical e as novas perspetivas, nas quais as fronteiras entre a filosofia da música e a estética da música, a musicologia e as abordagens das ciências sociais são cada vez mais frágeis. Como abordagem fundamental, defende-se que os temas e preocupações que aparecem na literatura são atualizações de problemas antigos, antes geralmente assumidos da racionalidade formalista, de maior tradição do que os pressupostos mais fenomenológicos e hermenêuticos.

Palavras-chave: filosofia da música, formalismo, significado musical, música culta

\section{Music and Philosophy (of Music): State of the Question. A Review of Problems, Discussions and Perspectives}

\begin{abstract}
This work investigates the main problems, discussions and perspectives in which the question of the philosophy of music is currently shaped. It is based on a review of articles published in journals of high impact on the international scene, recovered through the Scopus and Web of Science databases. The analyses are complemented, at times and according to the criterion of relevance, with other publications. The results suggest a field in expansion and specialization that crosses the transition between what we could call the classical perspective of approach to the musical object and the new perspectives, where the frontiers are increasingly fragile between philosophy of music and aesthetics of music, musicology and approaches of the social sciences. As a fundamental approach, it is defended that the themes and concerns that appear in literature are updates of old problems, usually assumed
\end{abstract}


before from formalist rationality, of greater tradition than the more phenomenological and hermeneutic assumptions.

Keywords: Philosophy of music, formalism, musical meaning, art music

\section{Introducción: las herencias de la discusión}

A comienzos del siglo pasado hubo una serie de circunstancias que estallaron los campos, relativamente estables, de la estética (general y musical), la filosofía de la música y la musicología. Factores externos (la masificación de la música popular, parte de un movimiento más general de desregulación de la cultura en su paso a los juegos de mercado) e internos (en lo académico, división de campos de estudio, respuesta a su hiperespecialización; en la creación, el impacto mayor de las vanguardias con la denominada nueva música) agitaron las aguas sobre las que fluían con relativa tranquilidad los debates de la tradición.

Para comienzos de siglo, la tendencia del análisis formal, liderada por Hanslick, se presentaba como un polo opuesto a la metafísica de Schopenhauer: los debates iban entre la pregunta por la forma musical y su esencia. Es sobre ese debate que el siglo XX deja nombres como Adorno, Wittgenstein y Jankélévitch, protagonistas de una discusión que, algunas veces, se trató de entender como la oscilación entre la dialéctica del arte musicológico, la filosofía analítica y la filosofía continental, con todo y las imprecisiones propias de estas denominaciones. Lo que permiten ver estas etiquetas es una preocupación por comprender la música desde su esencia, desde su contexto y desde su condición inefable, en el trasfondo de la indagación sobre su significado, que en el formalismo se trató de inspeccionar mediante la aplicación de métodos racionalistas (basados en modelos lingüísticos), y que, del lado opuesto, se trató de formular en una perspectiva post-analítica de corte fenomenológico.

Así, en tanto "reflexión de segundo nivel sobre la naturaleza de la música y nuestra experiencia de ella" (Marrades Millet, 2012, p. 5), la filosofía estuvo interesada en el siglo pasado por los siguientes problemas: metodológicos (formas de investigación de lo musical, concepciones y definiciones del fenómeno), ontológicos (formalismo, realismo, nominalismo, idealismo...), estéticos (propiedades y distinciones asociadas a la dimensión sensible de la música), semánticos (significado de la música), asociados a la experiencia musical (asuntos psicológicos, cognitivos, morales; expresividad, respuestas y conductas asociadas a la música) y axiológicos (qué hace valiosa a la música). 
Si se mira en el horizonte de la tradición, estos campos surgen en el debate de viejos problemas, donde se ha tejido la contextura que hoy tejen en el campo de la filosofía de la música. Fue en el terreno propio de la argumentación filosófica donde ocurrió la emergencia de los primeros campos de estudio: la filosofía de la música (Beitia Bastida, 2017; Rowell, 1996), la estética de la música (Fubini, 1999) y la musicología (Schiavio, 2012), si bien hacia las últimas décadas del siglo anterior estos se ampliaron en reflexiones de otro corte.

Esa re-partición de campos surgió debido a la hiper-especialización en las perspectivas de abordaje (Fubini, 1999), lo cual dificulta su visión de conjunto. Sin embargo, se puede establecer que la filosofía de la música discute asuntos de carácter ontológico, la estética musical los temas asociados a las experiencias sensibles de la música, y la musicología se centra en su análisis formal. En una visión sintética, la filosofía problematiza la música, la estética estudia la musicalidad y la musicología analiza lo musical.

Cuando se mira desde la filosofía de la música, la definición un tanto ambivalente de la antigüedad opera como barrera que lleva a asumirla, a veces, como sinónimo de pensamiento (Gallope, 2017; Levinson, 2009; Tarasti, 2008), otras como asunto sensitivo (Bidon-Chanal, 2015). Cuando se la plantea en los primeros términos, la vía racionalista apuntalaba un proyecto que se tradujo en el formalismo, que asumió su tarea moldeándola en categorías como autor, composición y estructura, asunto que lleva implícitas las nociones escriturales de la filosofía y las resonancias lingüísticas del decir. Cuando se lo mira desde la estética musical, la musicalidad prefigura como una condición sensible tejida en la intencionalidad expresiva del creador y la experiencia sensible del oyente (Matherne, 2014; Borio, 2009). Siendo un asunto que se configura en los horizontes de elaboración de sentido de ambas subjetividades, la musicalidad surge como proceso hermenéutico (Pontara, 2015; Barbu-Iuraşcu, 2008a) que no necesariamente pasa por la racionalidad, si bien puede incluirla. Es de resaltar que el problema del significado ha sido de interés en la discusión porque ha llevado a problematizar los términos que cada campo de estudio ha empleado para entender su esencia y su contexto (Whale, 2015; Pontara, 2015; Negretto, 2012; Oltețeanu, 2009; Tarasti, 2008; Bar-Elli, 2006; Scruton, 1987).

Así, dos perspectivas pueden acuñarse como las que han desarrollado el estado de la cuestión: el racionalismo formalista (aproximación estética clásica y trascendente) y la fenomenología hermenéutica (aproximación inmanente). Vistas en la perspectiva del tiempo, si la primera hace caso 
de la polaridad apolínea y la definición de música como pensamiento, la segunda abre la posibilidad de lo apolíneo y permite asumir la música como expresión, inspiración, sensitividad y encuentro (Martinelli, 2019). Es decir, si los intereses de la esencia se han centrado en lo apolíneo, los intereses por el contexto han privilegiado el entendimiento de la música como experiencia. En efecto, la recategorización de asuntos que propone Marrades Millet (2012) es la modulación que logró la tradición, a partir de unas tensiones aparecidas ya en los griegos. Siendo que desde Platón se abordó la cuestión de la música a través de nociones ontológicas, cosmológicas y pedagógicas (García Peña, 2013), a más se intentaron zanjar las discusiones, y al no reconocer la ambivalencia a la que invitaba la idea misma de música, la racionalidad ideó un camino que, buscando agotar el objeto, no hizo otra cosa que atomizarlo en las áreas y campos señalados por Fubini (1999).

Hoy parece que las incursiones neurocientíficas son la parte más sofisticada de ese esfuerzo con el que una significativa parte de la academia ha pretendido racionalizar (Barker, 2010) y cientifizar (Brioschi, 2016) los numerosos mitos asociados a la música (Butler, 2015; Berns and Moore, 2011; Levitin, 2018; 2019); a pesar de estos empeños, el residuo mítico no se ha logrado desvanecer satisfactoriamente (Kozel, 2016). No parece que sin asumir esa ambivalencia:

Una región media entre la mirada ya codificada y el conocimiento reflexivo, que es la más fundamental: anterior a las palabras, a las percepciones, a los gestos, que la traducen con mayor o menor exactitud. Es una región más sólida, más arcaica, menos dudosa, siempre más "verdadera" que las teorías que intentan darle una forma explícita, una aplicación exhaustiva o un fundamento filosófico (Beitia Bastida, 2017, p. 322).

La cuestión ya estaba servida siglos atrás: el arquetipo dionisíaco (flautista), versus el apolíneo (lirista) asoman una numinosidad en la que se centra la cuestión entre lo sensual y lo racional (Distaso, 2009; Fubini, 1999), que se puede traducir en términos de inmanencia y trascendencia. Tocando la flauta, Dionisio invita al baile (sensual, inmediato) que no permite la palabra -logos-; dotado de lira, Apolo propone la vinculación a la música desde la palabra, trascendente, lógica, racional. Dado que lo racional predominó, se pretendió entonces que música y filosofía nacieran con orígenes comunes: el logos.

Esta cuestión fondea en la distancia supuesta entre las apariencias y las ideas (Jonas De Aguiar, 2017): entendiendo las primeras como fuente de 
ilusión y engaño, la filosofía buscó asegurarse, "lo que contaba, entonces, era la propia actitud de contemplación y olvido de sí mismo hacia una obra de arte, más que la obra misma" (Beitia Bastida, 2017, p. 306). Aún en la Edad Media esa tensión seguía figurando (Dyer, 2007).

En el marco del problema en términos del choque apariencia/significado, espíritu/materia, proyecciones de las polaridades apolínea/dionisíaca, se inscribió buena parte de la actitud como la filosofía enfrentaría en adelante el fenómeno musical (Kozel, 2016; Galparsoro, 2013; Sanguinetti, 2012). El estado de la cuestión presenta resonancias de ese planteamiento inicial. Por contra, hoy urge una argumentación que amplíe horizontes de interpretación de la música como hecho social. El legado de la tradición, volcado al tamiz formalista racional, impide asir todas las formas de música que existen en la actualidad. Las más depuradas herramientas del formalismo musicológico impiden acotar la diversidad de contextos y formas, no solo por el hecho de que por fuera de las partituras se vive y se crea música, sino porque sin reconocer la música como fuente de placer es imposible comprender filosóficamente su imagen completa.

No extraña que la filosofía tomara el camino racional, renunciando a lo sensitivo; pero esto dificulta elegir un marco comprensivo para leer hoy la música (Fubini, 1970): si de un lado aparece con intensidad la desconfianza por lo sensitivo (Jonas De Aguiar, 2017), del otro fulgura una invitación a reconocerlo (Currie y Killin, 2016). Esto supone una dificultad, pues a la desconfianza por lo sensitivo deben sumarse la ignorancia sobre lo sonoro (Trías, 2010; Trías, 2007) y la exagerada visualidad de la episteme occidental (Galparsoro, 2013).

Lo vemos en la relación música-palabra (Pinilla Burgos, 2005). Si en el Romanticismo ocurre el triunfo de la música (pura), esta victoria sondea el triunfo de la razón y la abstracción (Martinelli, 2019; Bonds, 2014; Sanguinetti, 2012). Pero allí se entregó un lugar al arte, a la música y a los creadores donde la numinosidad recibía atención (Blanning, 2011; Steinberg, 2008). Esto es fundamental para la filosofía de la música del siglo anterior pues la música-filosofía produjo allí una fusión de músicapensamiento sin precedentes que puede entenderse como una inflexión de la ambivalencia Apolo/Dionisio.

Al llevar al máximo nivel la expresión y la importancia musical, se aumentaba la urgencia de resolver filosóficamente los asuntos pendientes. Una reacción lo intentó vía logos racional (desde el formalismo), con pretensión de agotar el significado de la música (Oyola Pérez y Borges, 2010; Tarasti, 
2008; Walser, 2003); otra, procuró creer la importancia de la música no limitada a la forma musical, si bien pasaba por ella (Kozel, 2016); otra más, postuló el reconocimiento del puro-sonido (Stratilková, 2016; Moreno, 2012) como vía para acercar lo expresivo (Barbu-Iuraşcu, 2008a; 2008b) y lo emocional (Stratilková, 2016), como sensibilidades que sobrevienen la experiencia musical (Marín, 2014; Martínez y Epele, 2012; Moreno, 2012; Bermúdez Rey, 2003).

En lo que sigue, se intenta señalar los principales puntos de tensión en la problematización de la música. Se muestra, primero, la vía racional, dialéctica y formalista, que surge del intento de dirigir por un mismo camino el proyecto de resolver su ambivalencia; segundo, la vía fenomenológica/ hermenéutica, la cual trata, de alguna forma, de hacer trámite a dicha ambivalencia.

\section{La tradición racionalista/dialéctica}

La tradición racionalista es la más ampliamente desarrollada, incluso en el Romanticismo que, invitado por Nietzsche, otorgó un lugar a lo mítico (Galparsoro, 2013; Trías, 2010). En Adorno se aprecia el paisaje divisado desde allí: "Un creciente cuerpo de opinión establecida ha llegado a reconocer a Theodor Adorno como el apóstol y apologista de la música de vanguardia, como el mayor oponente de la teoría de la reflexión y, en consecuencia, como el crítico que más ha hecho para 'aflojar' la relación causal entre el arte y la sociedad" (Feher y Feher, 1975, p. 99). ${ }^{2}$

En Adorno se observa el esfuerzo intelectual para establecer límites entre música culta y popular, reubicar el lugar de la obra desde la dialéctica negativa y defender la totalidad, orgánica y desconectada de otros fines distintos a la música misma, como lugar de liberación, racional e ilustrada del sujeto (Martinelli, 2019; Beitia Bastida, 2015). Esto lo pone como un parteaguas de la filosofía de la música: Adorno ratifica desatender lo sensible en favor de lo intelectual, cuando lo otro de la música culta eran las músicas populares. Pero a partir de las posguerras, un auge inesperado e incómodo agitó las aguas que antes solo movían las discusiones formales (Martinelli, 2019; Gallope, 2017; Fischerman, 2011): la explosión de la

\footnotetext{
${ }^{2}$ Todas las traducciones son propias. En adelante, solo transcribiremos el texto original en pie de página, sin advertirlo. "An increasing body of established opinion has come to recognize Theodor Adorno as the apostle and apologist of 'avant-garde' music, as the major opponent of the reflection theory, and consequently as the critic who has done most to 'loosen up' the causal relationship between art and society".
} 
música popular, transitando a lo masivo, implicó un denuedo intelectual para Adorno, uno de los primeros pensadores que hubo de ilustrar la actitud como la élite cultural debía frenar el impulso populoso y vulgar de las músicas emergentes. Esta misión se complicó por la crisis vivida por la música culta, vía vanguardias, y por la evidencia irrebatible de que el mundo cultural viraba hacia otros intereses.

Adorno es un bastión incuestionable de la perspectiva racionalista-dialéctica. Esto se debe a su postura radical respecto al lugar del arte como plataforma liberadora del individuo:

[...] la obra de arte exige una mediación subjetiva en su constitución objetiva, ya que la obra no se puede separar de la expresión de un sujeto. En esto consiste la espiritualidad del arte. Adorno considera que la participación subjetiva en el arte forma parte de su objetividad, puesto que en el arte es indispensable que se produzca lo que él llama el momento mimético, que se obtiene mediante lo idiosincrásico de los sujetos individuales (Bautista García, 2013, p. 308).

Comprometido con la mediación sujeto/obra, este pensamiento acusará, allí donde crea verlos, cualesquier coqueteos de lo fácil en lo culto. Adorno suscribe en la música la necesidad del reconocimiento de la polaridad apolínea/dionisiaca, pero condiciona: "Siempre que su sosiego parece perturbado por emociones básicas, se habla de la decadencia del gusto" (Adorno, 2009, p. 15). Esta actitud recia se aplica incluso contra compositores cultos que se dejen llevar por impulsos simplistas, donde la sencillez no es modo de expresión del carácter subjetivo del compositor sino complacencia, cliché que ambiciona atrapar la atención del oyente: "[Adorno] ya nos advirtió sobre la peligrosidad de vincular a Beethoven, Brahms y otros compositores con melodías famosas e ingenuas compuestas por ellos, argumentando que esos pasajes no son más que ruinas de su música y ya no tienen el mismo significado profundo que tenían cuando eran entendidos como partes de piezas mucho más grandes" (Jonas De Aguiar, 2017, p. 14). ${ }^{3}$

Esto ocurre porque su herencia dialéctica hegeliana del arte encomendaba la relación sujeto-objeto; el primer polo encara lo espiritual, el segundo lo material (Sanguinetti, 2012; Feher y Feher, 1975). Esta es la razón por la

\footnotetext{
3 “[A]lready warned us about the dangerousness of linking Beethoven, Brahms and other composers to famous and naïve melodies composed by them, arguing that those passages are but ruins of their music and have no longer the same deep meaning they had when they were understood as parts of much bigger pieces".
} 
cual, como mímesis, la obra es una entidad que existe por sí misma (Welten, 2009), que refleja, habrá que ver en qué formas, la idea del autor. En ese sentido, la obra ocurre por una totalidad, un principio en el cual, dialécticamente, compositor y obra marcan las relaciones internas estructurantes del proceso de movimientos, juegos de tensiones y distensiones desde los cuales puede mediarse la libertad individual como triunfo del espíritu sobre la materia sonoro-musical. Efervescente, esta totalidad surge con un orden interno; donde no prorrumpa esa totalidad orgánica por tal impulso interno, surgido por voluntad del creador, Adorno desdeña el detrimento del ejercicio dialéctico de la razón creadora, en favor de factores-otros que reemplazan la voluntad del individuo racional. Da igual si se trata de serialismo, expresionismo o jazz: donde el azar se regodea desaparece el dominio creador; cuando ese dominio descansa en una voluntad externa al oyente, ocurre la alienación (Leslie y Rantisi, 2019; Stratilková, 2016; D. Martinelli, 2008).

Ante estas formas musicales, Adorno enrostra la peligrosa orientación hacia la configuración de imágenes, inorgánicas, caleidoscópicas y fragmentarias que deslíen el logos discursivo-racional. La cuestión revalida la desconfianza de ese logos-racional hacia lo visual, lo emotivo y lo placentero (Pogăceanu, 2008).

En términos dialécticos, la negatividad del arte es la negación que la obra opera sobre el mundo. Para Adorno "La experiencia negativa, es decir, el carácter crítico de la obra de arte respecto de una sociedad determinada (era) el principal aporte de la obra de arte y [en ella] veía la expresión más radical de esta en el arte moderno" (Bidon-Chanal, 2015, p. 202); así, el arte debe ser negativo siempre que allí entendamos contra-posición al estado de cosas del mundo. Siendo un asunto amparado en la subjetividad ilustrada, el compositor plantea una negatividad en el proceso de predisponer la totalidad de la obra, que el oyente ha de positivar.

En esa visión adorniana se operó una mirada extrema, más bien cercana a lo aristocrático, con desconfianza por el gusto (fácil) de las masas. Por eso, a pesar de la gran atracción que ejerció en su momento Adorno, cada vez se dificultaba invitarlo para revisar los fenómenos cuya velocidad de aparición/desaparición crecían (Fubini, 2004). El encomiado esfuerzo invertido por Adorno, se lee hoy como un ejercicio aristocrático que inspiraba agudas líneas que no podían esconder la desconfianza por la personas no preparadas para asimilar la alta cultura, como se aprecia en su clasificación de los oyentes: el buen oyente, el consumidor de cultura, el emocional y el resentido (Adorno, 2009). Una lectura así dificulta 
comprender la cuestión musical; pero no pasa inadvertida su influencia al plantear cuestiones fundamentales en la reflexión musical (Kanellopoulos, 2012; Borio, 2009; Pogăceanu, 2009).

Adorno no es el único racionalista, pero es un representante sustancial, que sirve como ilustración del peligro que se advierte en la vinculación del logos musical con el logos-razón (Trías, 2010), adscripción que tiende a agotar el papel de la música como estancia para afirmar cosas del mundo; es cierto que Adorno defendió una idea de música en la que el significado es interno y no se refiere a cosas "objetivas del mundo"; pero también es cierto que al proponer la razón como pre-requisito del disfrute musical, dejó por fuera otras alternativas de nexo con la música.

Por más que se admita que el significado surge orgánicamente en la totalidad de la obra, en esa afirmación hay una imposición, pues así la músca tendría que revelar una equivalencia entre el sonido y las cosas del mundo (Martinelli, 2019). En uno u otro caso, el signo musical es portador de ideas que lo trascienden; allí reside una razón que debilita la vía racionalista:

Uno de los grandes problemas de la filosofía de la música es que, aunque intuitivamente la música parece ser significativa (a veces incluso de significado "profundo" e "iluminador"), parece ser particularmente terca y resistente a cualquier tratamiento "semántico": Siempre ha sido difícil ver cómo se puede atribuir "significado" a la música de manera fructífera, es decir, de una manera que realce una teoría de la comprensión de la música, de manera similar a la forma en que esta noción se aplica al lenguaje (Bar-Elli, 2006, p. 222). ${ }^{4}$

Una tal equivalencia no podría entenderse de otra forma que no fuera la representación. La música ha de representar, decir algo sobre el mundo: afirmar. Al contrario, algo común al serialismo y al expresionismo es que los sonidos no aparecen ligados a una referencia distinta que ellos mismos. El jazz puede ser clave para comprender otras formas de significación de la música: la alusión y el instante. La experiencia del jazz produce fragmentos musicales donde los sonidos no se anclan a representaciones sino que aluden: en el tono del instrumento, la propia voz del instrumentista, en los fraseos, su forma de entablar conversación con su

\footnotetext{
4 "One of the great problems in the philosophy of music is that, although intuitively music seems to be meaningful (sometimes even of "deep" and "illuminating" meaning), it seems to be particularly stubborn and resistant to any "semantic" treatment: It has always been difficult to see how "meaning" could be fruitfully ascribed to music, i.e. in a way that enhances a theory of understanding music, in anything like the way that this notion is applied to language".
} 
tradición (citas, menciones, variaciones de otros fraseos de otros solistas) (Murray, 2007). Es desde ahí desde donde el jazz permite expresar y sentir emociones.

Para la racionalidad apolínea, la noción de instante destruye la totalidad y enarbola el fragmento discontinuo mediante la acrobacia del solista, por encima de la logicidad y la totalidad orgánicas, caras a Adorno (Adorno, 2009). Sin embargo, siempre que se acuse fragmentación o asistematicidad, es probable que estemos ante principios organizadores o expresivos que se salen de nuestros marcos comprensivos desde los cuales entablamos las expectativas.

Representante del racionalismo que acusó la decadencia de la música, la reacción de Adorno contra el jazz, el serialismo y expresionismo evidencian una demanda que ya la música no podía cumplir: representar por vías del logos-lingüístico, controlar el acto creativo como instancia de erección del ego, asumir lo sensitivo como culpa.

Formas alternativas de comprender lo musical surgen por vías estéticas, fenomenológicas y hermenéuticas contemporáneas, al abrir nuevos canales auditivos para transitar la experiencia musical. Es difícil acogerlas como tradición; más que un cuerpo sólido de conceptos, son campos emergentes de discusión desde los cuales se propone un contrapeso a la tradición de los siglos anteriores (racionalista, formalista):

Tradicionalmente, los "puristas" o "formalistas" musicales han tratado de identificar lo musicalmente significativo con lo puramente musical. En los últimos tiempos, los teóricos, especialmente los influidos por ciertos escritos posmodernistas y el énfasis contemporáneo en la diferencia y el pluralismo, han insistido en que el significado musical sólo puede entenderse en el contexto institucional y social en el que se crean, presentan y disfrutan las obras musicales (Alperson, 1994, p. 8). ${ }^{5}$

\section{En busca de la experiencia: estéticas-otras, fenomenología y hermenéutica}

\footnotetext{
5 "Traditionally, musical "purists" or "formalists" have sought to identify the musically significant with the purely musical. In recent times, theorists, especially those influenced by certain postmodernist writings and contemporary emphases on difference and pluralism, have insisted that musical meaning can only be understood in the institutional and societal context in which musical works are created, presented, and enjoyed".
} 
El problema de la semanticidad de la música se discute en otros términos desde la experiencia musical, que en términos empíricos permite reconocer un compositor que expresa y un oyente que siente. Esa textura implica entonces que si la música produce emociones "una pieza musical expresa una emoción solo si esa emoción se despierta en el oyente" (Matherne, 2014, p. 139), ${ }^{6}$ es porque ella representa emociones, y desde esta constatación ampliar las nociones de significación: abrir otras acepciones más allá de las formales.

Basados en el jazz y algunas vanguardias, alusión es una idea que demanda otro tipo de aproximación a la música: el instante no se toma como evasión del discurso, descarga a la música de la diégesis (Rings, 2012). El contorno de la pregunta por la significación se desplaza de sus términos.

Esto implica una reubicación ontológica: la música "no es un decir, sino un hacer" (Jankélévitch, 2005, p. 127), más cerca del gesto que de la descripción analítica. Entonces, si una definición era la que permitía poner la atención en lo enunciativo, centrado en la composición y amarrando la significación a la capacidad de la música para referir cosas del compositor y del mundo, ahora pasamos a una idea en la cual la música es un suceder-se que alude (Szabados, 2014), cuyo discurrir media episodios significativos y emocionales.

En la literatura reciente, la figura de Jankélévitch, con resonancias del segundo Wittgenstein (Rings, 2012), permite ese acercamiento en clave inmanente que se convierte en inefable (Gallope et al., 2012; Hepokoski, 2012; Lochhead, 2012; Puri, 2012; J. Currie, 2012; Trías, 2010; Pogăceanu, 2009). Sí hay significado, pero opera por una experiencia que comprenden quienes participan en la conversación musical, no por un marco de referencia al estilo del diccionario. Así, la composición sí tiene esa direccionalidad exigida por el formalismo, pero es en el marco de la experiencia musical que esta adquiere, no ya significado, sino sentido (Freer, 2013; Rosales Meana, 2013).

Es Trías $(2007,2010)$ uno de los filósofos más sagaces en deshacerse de la idea de significado musical y pensar el sentido. Este, hecho de la numinosidad de la imagen, el mito y el silencio, alude a sensaciones que, en el plano de la duración, hablan de la inmanencia del estar en el mundo envueltos por su devenir. Llevar la discusión del significado al sentido es acoger la noción de mundo como aquel fragmento de las cosas del universo que entran en

6 "a piece of music expresses an emotion only if that emotion is aroused in the listener". 
el dominio humano; la música marca el límite del mundo en lo temporal, la arquitectura lo hace en lo espacial. Música y arquitectura conforman la textura del límite del mundo: lo hacen comprensible. Se deduce con Trías que si el significado es transmisión de información a través del texto musical, el sentido es horizonte del mundo que se teje en la experiencia musical.

Contrario a la visión formalista, la música "no puede ser presentada sin la contaminación de conceptos aproximativos y equívocos, la reflexión, los fenómenos, el intelecto..." (Gallope et al., 2012, p. 238). ${ }^{7}$ Ella ocurre en el mundo, está contaminada de él porque es justo en la música que se bordea la experiencia-mundo.

Las ideas de Jankélévithc (Gallope, 2017; Beitia Bastida, 2015; Gallope et al., 2012) despuntan para abrir la inmanencia (Martinelli, 2019). Según él, la dominación trascendente emergía cuando se atajaba la música a los mecanismos del lenguaje. El lenguaje (verbal) entorpece la unión vital de la música con la experiencia humana (Szabados, 2014, p. 22). Por eso, en la idea de experiencia radica buena parte de las discusiones contemporáneas sobre filosofía de la música por vías hermenéuticas (Pontara, 2015; Borio, 2009) y fenomenológicas (Hui, 2013; Hepokoski, 2012; Currie, 2012; Hui, 2011; Welten, 2009).

Desatada de vínculos racionalistas, la música nos devuelve a la crudeza del sonido como sonido en sí mismo: "se entiende la diferencia entre escuchar sonidos como signos de cosas y escuchar sonidos en cuanto tales" (Stratilková, 2016, p. 215), ${ }^{8}$ esto permite la distinción entre oír los sonidos como signos de cosas y escucharlos como acontecimientos. Esto impulsa la comprensión significativa del arte como algo, semántico sí, pero por fuera de la significación lingüística (Cárdenas-Soler \& Martínez-Chaparro, 2015). Ese es el concepto de experiencia (Pogăceanu, 2008): siendo la música un asunto vital, al centrar el interés en la propia experiencia, las reflexiones lenguaje/significado musical se refrescan y permite desplazamientos fundamentales para la tesitura de la discusión. Del ejercicio, categorías centrales de la vía racionalista pasan a ocupar nuevos lugares. Sucede con las ideas de autor (Bartleet, 2009; de Aguilera et al., 2010; Leonhard, 2010; Marín, 2014), sonoridad (Aymerich Goyanes, 2016; Viñuela, 2010) y obra (Botstein, 2012; Brown, 2000; Gunkel, 2008; Guter, 2015; Milutis, 2008; Van Eecke, 2014; Wilson, 2014).

7 " [...] cannot be presented without the contamination of approximating and equivocal concepts, reflection, phenomena, intellect, the 'actual', the 'gnostic', etc."

8 "Here the distinction between hearing sounds as signs of things and hearing sounds as such is meant". 


\section{Textura de la experiencia}

La experiencia musical ratifica un encuentro, que en la literatura se inspecciona tanto desde lo fáctico, como en lo comunicativo, encuentro de dos subjetividades: "Cuando los oyentes se encuentran con la música, como lo harían con una persona, descubren activamente la otra cara de la sinfonía, la cara que no es meramente los sonidos de la música, sino que implica lo que puedo describir mejor como la preocupación de la música por sus sonidos" (Whale, 2015, p. 28). ${ }^{9}$ El encuentro prefigura el tránsito entre lo sonoro y lo musical. Para lograrlo, se requiere el reconocimiento estético de la música como música (Marín, 2014, p. 113).

Ese encuentro suscita la posibilidad del significado que se da en el reconocimiento, estético y comunicativo, a través de la música:

Si hay un significado musical, es porque hay un entendimiento musical. Las teorías actuales de la música tienden a equivocarse, ya sea porque descuidan este simple pensamiento, o porque describen la comprensión musical en términos de alguna teoría cuya aplicación primaria es en un campo (como la lingüística) que no tiene nada que ver con la música (Scruton, 1987, p. 170). ${ }^{10}$

En cuanto a dónde reside el significado, los absolutistas afirman que el significado de una obra musical se encuentra dentro de la obra misma, mientras que los referencialistas enfatizan el significado de una obra que puede encontrarse en aquello no-musical a lo que la obra hace referencia; por otro lado, en cuanto a la recepción de ese significado por parte del oyente, los formalistas aseguran que el significado es captado intelectualmente entendiendo las relaciones entre los elementos musicales que constituyen la obra, y los expresionistas aseveran que las reacciones del oyente ante la música son de tipo emocional (Bermúdez Rey, 2003, p. 35).

Encuentro implica aceptar que nuestra noción de significado afecta lo que entendemos por música. Pero ni la expectativa frente a la noción de

\footnotetext{
9 "When listeners encounter the music, as they would encounter a person, they actively discover the other face of the symphony, the face that is not merely the music's sounds but involves what I can best describe as the music's concern for its sounds".

10 "If there is musical meaning, it is because there is a musical understanding. Current theories of music tend to go wrong either because they neglect this simple thought, or because they describe musical understanding in terms of some theory whose primary application is in a field (such linguistics) which has nothing to do with music".
} 
significado ni la experiencia musical misma ocurren en blanco; el contexto cultural acumula esquemas que moldean la experiencia interpretativa de la música; esta depende de la tradición desde la cual escuchamos, asunto que aleja la centralidad en el compositor de la tradición racionalista:

En otras palabras, la música no debe interpretarse apegándose ciegamente a la notación, es decir, a los signos visuales de la música, sino más bien se debe tratar de alcanzar las realidades conceptuales, estéticas, axiológicas y epistémicas, que se esconden detrás de esos signos visuales. Al igual que las demás artes, la música es inseparable de estos constructos culturales cognoscitivos (Tarasti, 2008, p. 20).

Es sobre el recorrido de experiencias personales moldeadas por lo social desde donde se configuran el significado y las expectativas respecto a la música: un proceso negociado, construido y emergente. El significado musical no trata de la transmisión de unidades discretas de información; su sentido (dirección) ocurre cuando los escuchas, a través de organizaciones perceptivas y formales, comprenden secuencias de sonido como música. Pero no es un asunto limitado a lo sonoro: "Ha habido muchos intentos de definir lo que es la música en términos de los atributos específicos de los sonidos musicales [...] no es posible llegar a una definición satisfactoria de la música simplemente en términos de sonido" (Cook, 1990, pp. 10-11). ${ }^{11}$

¿Cómo entonces arrostrar esa materialidad que, siendo física, no es únicamente material? En otras palabras, ¿cómo emerge el tránsito entre sonido y música? La vía fenomenológica propone la experiencia material (sonora) y la memoria, como los lugares donde se concreta la amplitud de las referencias y esquemas musicales desde los que cada persona se relaciona con ella. La suma de experiencia sensible y memoria propician la experiencia sensible, otorgan significado en el mundo. El objeto musical deviene tal en ese proceso: "Como sujeto no puedo experimentar el mundo. Lo que se experimenta es el significado intencional del mundo, mientras que el mundo mismo es el objeto pretendido, que es trascendental por definición" (Schiavio, 2012, p. 69). ${ }^{12}$

\footnotetext{
11 "There have been many attempts to define what music is in terms of the specific attributes of musical sounds [...] it is not possible to arrive at a satisfactory definition of music simply in terms of sound".

12 "As a subject I cannot experience the world. What is experienced is the intentional meaning of the world, while the world itself is the object intended, which is transcendental by definition".
} 
Se requiere entonces entender que los significados son atribuidos, imputados, no preexistentes (Bar-Elli, 2006). Más que representar, la música alude. Para ello se precisa dejarse llevar por la experiencia de la música: aún en el contexto del lenguaje verbal las palabras buscan arrastrar a una experiencia compartida de sentido.

Así, el hacer del que hablara Jankélévitch surge por una invitación a compartir la experiencia vital. No se trata de una experiencia meramente psicológica o exclusivamente racional; es una experiencia vivaz que se percibe, fluida, en la música. En tal experiencia, lo que procura la relación sincrónica entre la música y el mundo se gesta desde la ondulación propia del sonido por cuanto esta evidencia el palpitar mismo del cosmos (Bar-Elli, 2006).

Tal como el mundo deviene movimiento, el sonido surge como evidencia del movimiento mismo de la vida. Buscar orientaciones de lo musical no debe convertirse en estrechar la experiencia a un proceso de transmisión informativa: la música se refiere a sí misma. Ante esa sutileza de la configuración significativa entre compositor y oyente, cabe la posibilidad de que la verbalización del hecho musical desprenda la naturalidad con la que ocurre el hecho musical. No se debiera hablar de música sin considerar que ella nos propone una conexión directa con la vida: "al escuchar música, podemos imaginarnos introspectando o simplemente experimentando sentimientos, y esto de una manera musical" (Alperson, 1994, p. 3). ${ }^{13}$ Contra dicha experiencia, hablar/escribir de música erige postura crítica, distancia (Pontara, 2015): si la música es posible con tanta desenvoltura, la filosofía de la música reingresa al decir lo que, en la vida, es un hacer.

Contrario a la experiencia reflexiva, el sentir humano se encuentra diluido, agitado o apaciguado en su encuentro con la música; pensar lo musical ayuda a relevar el asunto emocional que media la música (Michels-Ratliff \& Ennis, 2016). Cabe preguntarse entonces cómo ocurre esa mediación a través de lo sensible, comprender la experiencia musical como un escenario de configuración imbricada de sentimientos (feelings), emociones (emotions) y afecciones (affections). En la emoción hay un estado anímico de base que se desarrolla a través del juego de expectativas y anticipaciones que el oyente establece con la música. La expectación "puede considerarse como una estrategia básica de la mente humana que refleja una tendencia, un movimiento intencional hacia el futuro" (Negretto, 2012, p. 151); por su parte, la anticipación es "un estado mental de suspenso sobre

13 “[...] in listening to music, we can imagine introspecting or simply experiencing feelings, and this in a musical way". 
lo que está pasando, durante el cual se espera que ocurran una serie de eventos probables" (Negretto, 2012, p. 154). Ambos son dialécticamente decisivos, en tanto "la anticipación es un momento particular durante el proceso de expectativa: es el momento en el que los oyentes representan en su mente precisamente cómo la música continuará" (Negretto, 2012, p. 154). ${ }^{14}$ Las emociones se suscitan cuando la música entrega algo diferente de lo que se había anticipado.

La afección tiene que ver más con cómo lo que se percibe afecta el estado de ánimo (Shouse, 2005), mientras que los sentimientos surgen como emociones y afecciones que en la experiencia vital (y musical) el sujeto modula con mayor claridad. Se crea así una suerte de enciclopedia con convenciones emocionales, más o menos vinculadas a estilos musicales o a estilos particulares de los compositores y que se identifican en los registros de la crítica musical, las dataciones y las periodizaciones de que son capaces la historiografía y la musicología (Mueller, 2012). En esos bancos de memoria, la esfera individual negocia bagajes que en los entornos culturales son de dominio público.

La base del trámite interior de la música, entonces, ocurre por las emociones, desde las cuales la persona estructura, acomoda y organiza esquemas de interpretación y organización del significado. La música funge motor y espejo de las emociones (Marín, 2014); opera como nodos de vivencia sobre los cuales se organiza la memoria musical en encuentro con la memoria personal y social. Por tanto, más que instancias fijas, autor y oyente aparecen, en el seno de la experiencia, como motores y espejos emocionales. Ni el trámite emocional se define únicamente en la partitura o el canon de fórmulas y convenciones, ni la pura materialidad sonora alcanza ordena las relaciones dinámicas entre formas sonoras y estados emocionales que se desatan en las personas con la música.

En la estética de la expresividad se inspecciona este lugar como espacio de amplias posibilidades de sentido. En el límite de la interioridad, donde se despiertan las emociones gracias a los estímulos musicales y a los esquemas con los cuales esta experiencia se organiza, es donde ocurre la representación: "si podemos establecer que la música es a menudo emocionalmente expresiva y que a menudo despierta emoción, todo lo

\footnotetext{
${ }^{14}$ [Expectation] "may be considered as a basic strategy of the human mind that reflects a tendency, an intentional movement toward the future"; [anticipation] "a mental state of suspense about what is going on, during which a range of probable events are expected to happen". "Anticipation is a particular moment during the process of expectation: it is the moment in which listeners represent in their mind how precisely the music will go on".
} 
que queda es dar un análisis satisfactorio del concepto de representación" (Kim et al., 2010, p. 332). ${ }^{15}$ Esta representación se entiende como la concurrencia de tres condiciones: "la condición de contenido", "la condición de intencionalidad" y "la condición de éxito". ${ }^{16}$

Se ha discutido la configuración de estas tres condiciones en la música. Contenido es la disposición intencional de elementos sonoros, modulados mediante el lenguaje musical (armonía, ritmo, melodía, timbre) (Sanguinetti, 2012; Barker, 2010; Marius Varvaroi, 2010; Méndiz Noguero, 2010; Distaso, 2009); intencionalidad está en el uso de los recursos musicales para, en el juego de expectativas y anticipaciones, propiciar las emociones (Negretto, 2012); éxito se ratifica en el constante encuentro de los oyentes con la música y el impacto que esta promueve en las emociones.

Sin embargo, la hermenéutica ha ayudado a problematizar el asunto como una situación compleja. Si el contenido se asumió como instancia única del compositor, se problematizan los lugares del director (Bartleet, 2009) y del instrumentista (Kania, 2006) a la hora de definir el contenido de la obra. Pese a que una parte de la tradición sostiene que "la hisotria de la música es principalmente la hisotria de sus obras" (Olteţeanu, 2009, p. 162), a la luz de los avances tecnológicos aparecen nuevos problemas que cuestionan la obra como único lugar de desarrollo de esta historia, toda vez que no es la escritura el único lenguaje con que esta se fija.

La intencionalidad se ve sometida a constantes revisiones por vía de las experimentaciones vanguardistas que agitaron la supuesta direccionalidad de la composición al introducir elementos de azar (Bauer, 2010); también desde nociones como paisaje sonoro (Cárdenas-Soler \& Martínez-Chaparro, 2015; Viñuela, 2010; Woodside, 2008) donde la música es tan solo uno de los componentes que definen la sonoridad de la experiencia sensible contemporánea. Así, la producción del contenido emocional está contaminada por circunstancias, técnicas y humanas, que median entre la idea del autor y la percepción de la música.

Con todo, la fenomenología aporta la reflexión sobre la experiencia musical, la estética abre posibilidades de lectura de los intercambios sensibles y la hermenéutica se ocupa de los constantes juegos de interpretación que, de facto, definen la experiencia musical.

\footnotetext{
15 "If we can establish that music is often expressive of emotion and that it often arouses emotion, all that remains is to give a satisfactory analysis of the concept of representation".

16 "[T]he content condition", "the intentionality condition" y "the success condition".
} 


\section{Conclusiones}

A la luz de lo anterior, se aclara que la emergencia de campos de estudio y perspectivas no es otra cosa que el vaivén surgido por la acomodación entre concepciones de música, diseño de métodos para estudiarla y el asombro, siempre vigente, ante lo que las experiencias musicales generan en lo humano. Como contestación al racionalismo formal, las perspectivas fenomenológica, estética y hermenéutica claman por una mirada más anclada en los sujetos reales que disfrutan de la música. Si el análisis musicológico permitió acercarse a recursos, repertorios y técnicas de organización del material sonoro, descuidar el lado de la recepción, el disfrute y la experiencia, dejan incompleto el estudio de la música.

No extraña entonces que las categorías tradicionales del estudio racionalista y formal sean desplazadas de sus lugares convencionales. Autor, obra, significado no tienen por qué ser los portadores de la belleza musical, ni la belleza ha de ser el único tema de relevancia. Si en la apreciación musical juegan asuntos históricos y personales que contextualizan y actualizan lo que está escrito en la partitura, cabe sondear algunas de las consecuencias que esto trae para la tradición racionalista.

En efecto, una de las principales consecuencias parece ser la reelaboración de la idea de belleza. Esta idea había sido fundante de la apreciación antigua de la música, a la vez que estructurante de todo el camino de la filosofía, la estética y la música del romanticismo. Pero, además, fue uno de los moduladores principales desde los que se congeló el avance de la experimentación estética de la música. Acorde a Blanning (2011), Fischerman (2011) y Steinberg (2008), desde la conformación del canon la belleza jugaba como propuesta de articulación y selección de los repertorios, donde el circuito de conservatorios y salas de concierto fue depurando las obras en fragmentos y fue troquelando una idea de belleza asociada a estos fragmentos que resultaron privilegiados frente a otros. Sin duda, esa selección fue el paradigma desde el cual se juzgó la música que se siguió creando, de forma que algunos compositores optaron por complacer las expectativas del público, mientras otros prefirieron seguir su camino creativo en búsqueda de sus propias cotas.

Es indudable que así como sirvió para fijar tal idea de belleza, la estética, en tanto campo de estudio, problematizó esta noción misma, a punto de considerar que ella no está contenida en algunos atributos objetivos de las obras, sino que pasa por unos procesos de negociación entre espectadores y compositores en el contexto del entorno social e histórico (Barker, 2010; Levinson, 2012; Perman, 2011) 
En lugar de la belleza, la estética aplicada a estos emergentes productos culturales procura una ampliación de las concepciones de música (de música a músicas, se proclama), y las aproximaciones más desde las ciencias sociales (semiótica, antropología, sociología y comunicación) ${ }^{17}$ se interesan más por asuntos como la evocación de nostalgia (y emociones en general) y la configuración -e incluso la predicción- de la popularidad (Berns \& Moore, 2011).

Llama la atención que sean desarrollos tecnológicos los que empujen la reflexión ontológica sobre categorías convencionales como autor y obra. Es como si la irrupción de otro sistema de dispositivos obligara a pensar en nuevos términos viejas cuestiones. Pero también deja la sensación de que, ineludiblemente, lo que se ha pensado sobre música se estructura frente a unos modos particulares de la experiencia, en concreto, el concierto clásico, la música culta y la composición formal. Lo cual se pudo mantener relativamente estable entre el Romanticismo y el auge de las vanguardias del siglo XX. Luego del auge de la música popular masiva, la crisis de las formas clásicas de cultura y el paso de la cultura a juegos del mercado, las viejas seguridades racionalistas y formales quedaron suspendidas en su eficacia, cuestionadas en su fundamentación y evidenciadas en sus asunciones más elementales.

El camino que se sigue para la reflexión es, sin duda, uno en el que no se abriga ya la esperanza de una única verdad, unas pocas protoformas musicales y unos únicos dispositivos para mediar la música. Ahora que lo apolíneo se ha impuesto como fuerza social y que el mundo de la modernidad tardía se muestra menos capaz de contener los flujos de intercambio cultural dentro del contorno de unas formas tradicionales, es un gran momento para idear nuevos abordajes a un fenómeno que, desde la antigua tradición clásica griega, no ha dejado de cautivarnos.

\footnotetext{
${ }^{17}$ En la perspectiva semiótica, resaltan: Brioschi (2016), Cárdenas-Soler y Martínez-Chaparro (2015), Danesi (2008), Beeman (2006); en una perspectiva antropológica, ver: Hays y Lowenfels (2016), Mueller (2012), Kanellopoulos (2012); en una perspectiva sociológica, Gallego Pedraza (2010); en la perspectiva comunicativa, Ortiz Morales (2010).
} 


\section{Bibliografía}

» Adorno, T. (2009). Disonancias/Introducción a la sociología de la música. Madrid: Akal.

》 Alperson, P. (1994). New Directions in the Philosophy of Music. Journal of Aesthetics and Art Criticism, 52(1), 1-11. Recuperado de https://www.jstor. org/stable/431580

》 Aymerich Goyanes, G. (2016). Conectando energía sonora y visualidad. AusArt Journal for Research in Art, 4(1), 129-141. https://doi.org/10.1387/ ausart.16690

» Bar-Elli, G. (2006). Wittgenstein on the Experience of Meaning and the Meaning of Music. Philosophical Investigations, 29(3), 217-249. https://doi. org/10.1111/j.1467-9205.2006.00291.x

» Barbu-Iuraşcu, V. (2008a). Authentic Interpretation and Musical Impressions. Analysis \& Metaphysics, 7(1), 218-221.

» Barbu-Iuraşcu, V. (2008b). Musical Imagery, Harmonic Patterns, and Emotional Expression. Review of Contemporary Philosophy, 7(2), 270-273.

»Barker, A. (2010). Mathematical Beauty Made Audible: Musical Aesthetics in Ptolemy's Harmonics. Classical Philology, 105(4), 403-420.

» Bartleet, B.-L. (2009). Conductors and Authorship: A Postmodern Critique of Historical and Contemporary Perspectives. Limina. A journal of historical and cultural studies, 15(2), 1-10. Recuperado de http://hdl.handle. net/10072/30172

" Bauer, A. (2010). Philosophy Recomposed: Stanley Cavell and the Critique of New Music. Journal of Music Theory, 54(1), 75-90. https://doi. org/10.1215/00222909-2010-012

» Bautista García, V. E. (2013). Un concepto revisado de música clásica. Música oral del Sur: revista internacional, 1(10), 207-217.

» Beeman, W. O. (2006). Los tropos de la música. Revista de antropología social, 15, 265-271. Recuperado de https://www.redalyc.org/articulo. oa?id=83801511

» Beitia Bastida, M. Á. (2017). El lugar de la música en la filosofía del siglo XX. Endoxa, Series Filosóficas, 39, 305-325. Recuperado de http://e-spacio.uned. es/fez/view/bibliuned:Endoxa-2017-39-5070

» Beitia Bastida, M. Á. (2015). Las múltiples dimensiones de la música en el siglo XX. [UNED]. Recuperado de http://e-spacio.uned.es/fez/view/ tesisuned:Filosofia-Mabeitia

"Bermúdez Rey, J. P. (2003). Interrupción de tendencias y criterio del gusto: La estética del criterio del gusto de David Hume y realización en la filosofía de la música de Leonard Meyer. Universitas Philosophica, 40-41, 29-63. 
» Berns, G. S., \& Moore, S. E. (2011). A Neural Predictor of Cultural Popularity. Wiley Online Library, 22(1), 154-160. https://doi.org/10.1016/j. jcps.2011.05.001

»Bidon-Chanal, S. (2015). Música envejecida y retorno a la libertad. Las críticas a la Escuela de Darmstadt de TW Adorno y su propuesta de una "musique informelle". Cadernos De Filosofia Alemã: Crítica E Modernidade, 20(2), 201-217. https://doi.org/https://doi.org/10.11606/issn.2318-9800. v20i2p201-217

» Blanning, T. (2011). El triunfo de la música: Los compositores, los intérpretes y el público desde 1700 hasta la actualidad (1a ed.). Barcelona: Acantilado.

» Bonds, M. E. (2014). La música como pensamiento. El público y la música instrumental en la época de Beethoven (1a ed.). Barcelona: Acantilado.

"Borio, G. (2009). The Crisis of Musical Aesthetics in the 21st Century. Topoi, 28(2), 109-117. https://doi.org/10.1007/s11245-009-9054-7

" Botstein, L. (2012). Recording and Reality: The Musical Subject. The Musical Quarterly, 95(1), 1-14. https://doi.org/10.1093/musqtl/gds020

» Brioschi, M. R. (2016). Hints toward Cosmology: The Need for Cosmology in Peirce's Philosophy. Scio. Revista de filosofía, 12, 51-73.

" Brown, L. B. (2000). Phonography, Rock Records, and the Ontology of Recorded Music. The Journal of Aesthetics and Art Criticism, 58(4), 361-372.

» Butler, K. (2015). Myth, Science, and the Power of Music in the Early Decades of the Royal Society. Journal of The History of Ideas, 76(1), 47-68.

»Cárdenas-Soler, R. N., \& Martínez-Chaparro, D. (2015). El paisaje sonoro, una aproximación desde la semiótica. Revista Investigación, Desarrollo, Innovación, 5(2), 129-140. Recuperado de https://revistas.uptc.edu.co/ revistas/index.php/investigacion_duitama/article/view/3717

»Cook, N. (1990). Music, Imagination, and Culture. Oxford: Oxford University Press.

»Currie, A., \& Killin, A. (2016). Musical Pluralism and the Science of Music. European Journal for Philosophy of Science, 6(1), 9-30. https://doi. org/10.1007/s13194-015-0123-z

»Currie, J. (2012). Vladimir Jankélévitch's Philosophy of Music: Where Jankélévitch Cannot Speak. Journal of the American Musicological Society, 65(1), 247-251.

»Danesi, M. (2008). The Medium is the Sign: Was McLuhan a Semiotician? MediaTropes eJournal, 1, 113-126. Recuperado de www.mediatropes.com

»de Aguilera, M., Sedeño, A., \& Borges Rey, E. (Eds). (2010). Hibridando el saber: investigar sobre comunicación y música (1a ed.). Málaga: Universidad de Málaga.

> Distaso, L. V. (2009). On the Common Origin of Music and Philosophy: Plato, Nietzsche, and Benjamin. Topoi, 28(2), 137-142. https://doi.org/10.1007/ s11245-009-9058-3 
»Dyer, J. (2007). The Place of Musica in Medieval Classifications of Knowledge. Journal of Musicology, 24(1), 3-71. https://doi.org/10.1525/jm.2007.24.1.3

" Feher, F., \& Feher, Z. (1975). Negative Philosophy of Music. Positive Results. New German Critique, 4, 99-111.

» Fischerman, D. (2011). Después de la música. El siglo XX y más allá. (1a ed.). Buenos Aires: Cadencia Editora.

» Freer, A. (2013). Musicality and the Limits of Meaning in Wordsworth and Kant. Paragraph, 36(3), 324-343. https://doi.org/10.3366/para.2013.0097

» Fubini, E. (1970). Music Aesthetics and Philosophy. International Review of Music Aesthetics and Sociology, 1(1), 94-97.

» Fubini, E. (1999). La estética desde la Antigüedad hasta el siglo XX (7a ed.). Madrid: Alianza.

» Fubini, E. (2004). El siglo XX: entre música y filosofía (1a ed., Vol. 19). València: Universitat de València.

» Gallego Pedraza, I. (2010). Presencia e influencia del rock en la sociedad española del siglo XXI. En M. De Aguilera, A. Sedeño, \& E. Borges Rey (Eds.), Hibridando el saber. Investigar en comunicación y música (pp. 129-137). Málaga: Universidad de Málaga.

» Gallope, M. (2017). Deep Refrains: Music, Philosophy, and the Ineffable. (1a ed.). Chicago: Chicago University Press.

》 Gallope, M., Kane, B., Rings, S., Hepokoski, J., et al. (2012). Vladimir Jankélévitch's Philosophy of Music. Journal of the American Musicological Society, 65(1), 215-256. Recuperado de https://www.jstor.org/ stable/10.1525/jams.2012.65.1.215

»Galparsoro, J. I. (2013). Nietzsche y la cuestión de la primacía de lo visual en el pensamiento occidental. Contrastes. Revista Internacional de Filosofía, 19(1).

" García Peña, I. (2013). Cuatro sentidos de la música en la filosofía griega $=$ Four music senses in the Greek Philosophy. Azafea. Revista de Filosofía, 15, 21-37. Recuperado de https://www.academia.edu/ download/54700482/12250-44227-1-SM.pdf

"Gunkel, D. (2008). Rethinking the Digital Remix: Mash-ups and the Metaphysics of Sound Recording. Popular Music and Society, 31(4), 489-510. https://doi.org/10.1080/03007760802053211

" Guter, E. (2015). The Good, the Bad, and the Vacuous: Wittgenstein on Modern and Future Musics. Journal of Aesthetics and Art Criticism, 73(4), 425-439. https://doi.org/10.1111/jaac.12205

» Hays, L., \& Lowenfels, W. (2016). An Internationalist Culture of the Singing Left in the Twentieth Century. En Temple University Press (Ed.), We Shall Not Be Moved/No nos moverán: Biography of a Song of Struggle (1a ed., pp. 135146). Filadelfia: Temple University Press.

» Hepokoski, J. (2012). Vladimir Jankélévitch's Philosophy of Music: Ineffable 
Immersion: Contextualizing the Call for Silence. Journal of the American Musicological Society, 65(1), 223-230.

»Hui, A. (2013). Changeable Ears: Ernst Mach's and Max Planck's Studies of Accommodation in Hearing. Osiris, 28(1), 119-145. https://doi. org/10.1086/671366

» Hui, A. (2011). Instruments of Music, Instruments of Science:

Hermann von Helmholtz's Musical Practices, his Classicism, and his Beethoven Sonata. Annals of Science, 68(2), 149-177. https://doi.or $\mathrm{g} / 10.1080 / 00033790.2010 .519991$

» Jankélévitch, V. (2005). La música y lo inefable. Barcelona: Alpha Decay.

» Jonas De Aguiar, V. (2017). Ontological Incompleteness and Music by Slavoj Žižek. International Journal of Žižek, 3(11), 9-15. Recuperado de http:// zizekstudies.org/index.php/IJZS/article/view/1024

" Kanellopoulos, P. (2012). Envisioning Autonomy through Improvising and Composing: Castoriadis Visiting Creative Music Education Practice. Educational Philosophy and Theory, 44(2), 151-182. https://doi. org/10.1111/j.1469-5812.2010.00638.x

» Kim, Y. E., Schmidt, E. M., Migneco, R., Morton, B. G., Richardson, P., Scott, J., Speck, J. A., \& Turnbull, D. (2010). Music Emotion Recognition: A state of the Art Review. Proc. ismir, 86, 937-952.

"Kozel, D. (2016). Mythological Archetype in Music and Principles of its Interpretation. International Review of the Aesthetics and Sociology of Music, 47(1), 3-15.

» Leonhard, G. (2010). El precio de la libertad. Reinventando la economía online: Cómo los contenidos gratuitos pueden ser rentables a largo plazo. En M. De Aguilera, A. Sedeño, \& E. Borges Rey (Eds.), Hibridando el saber. Investigar en comunicación y música (1a ed., pp. 18-23). Málaga: Universidad de Málaga.

» Leslie, D., \& Rantisi, N. (2019). Deskilling in Cultural Industries: Corporatization, Standardization and the Erosion of Creativity at the Cirque du Soleil. Geoforum, 99, 257-266. https://doi.org/https://doi.org/10.1016/j. geoforum.2017.09.011

" Levinson, J. (2009). Philosophy and Music. Topoi, 28(2), 119-123. https:// doi.org/10.1007/s11245-009-9055-6

" Levinson, J. (2012). Musical Beauty. Teorema, 31(3), 127-135. Recuperado de https://www.jstor.org/stable/43046960

» Levitin, D. (2018). Tu cerebro y la música. El estudio científico de una obsesión humana. (1a ed.). Barcelona: RBA.

» Levitin, D. (2019). El cerebro musical (1a ed.). Barcelona: RBA.

» Lochhead, J. (2012). Vladimir Jankélévitch's Philosophy of Music: Can We Say What We Hear?-Jankélevitch and the Bergsonian Ineffable. Journal of the American Musicological Society, 65(1), 231-235. 
» Marín, U. (2014). Categorías filosóficas generales aplicadas a los fenómenos estéticos. Estudio de caso en la música y los discursos sonoros. CIENCIA ergo-sum, Revista Científica Multidisciplinaria de Prospectiva, 21(2), 112-120.

» Marius Varvaroi, C. (2010). Timbre musical. Evolución cultural y tecnología. En M. De Aguilera, A. Sedeño, \& E. Borges Rey (Eds.), Hibridando el saber. Investigar en comunicación y música (1a ed., pp. 159-165). Málaga: Universidad de Málaga.

»Marrades Millet, J. (2012). Una perspectiva sobre la filosofía de la música. Teorema: Revista Internacional de Filosofía, 31(3), 5-24.

»Martinelli, D. (2008). Music, Identity and the Strange Case of Authenticity. Musicology of Lithuania/Lietuvos muzikologija, 1(9), 122-130.

» Martinelli, R. (2019). Philosophy of Music: A History (1a ed.). Göttingen: Walter de Gruyter GmbH \& Co KG.

»Martínez, I. C., \& Epele, J. (2012). ¿Como se construye la experiencia intermodal del movimiento y la música en la danza? Relaciones de coherencia en la performance y en la recepción de frases de música y de movimiento. Cuadernos de Música, Artes Visuales y Artes Escénicas, 7(2), 6582.

» Matherne, S. (2014). Kant's Expressive Theory of Music. Journal of Aesthetics and Art Criticism, 72(2), 129-145. https://doi.org/10.1111/jaac.12076

»Méndiz Noguero, A. (2010). Filmografía mozartiana. Panorama histórico por las bandas sonoras con música de W. A. Mozart. En M. De Aguilera, A. Sedeño, \& E. Borges Rey (Eds.), Hibridando el saber. Investigar en comunicación y música (1a ed., pp. 24-36). Málaga: Universidad de Málaga. Recuperado de https://www.researchgate.net/publication/46308178

» Michels-Ratliff, E., \& Ennis, M. (2016). This is Your Song: Using Participants' Music Selections to Evoke Nostalgia and Autobiographical Memories Efficiently. Psychomusicology: Music, Mind, and Brain, 26(4), 379.

"Milutis, J. (2008). The Biography of the Sample: Notes on the Hidden Contexts of Acousmatic Art. Leonardo Music Journal, 18, 71-75. https://doi. org/10.1162/lmj.2008.18.71

"Moreno, C. (2012). Los oídos prestados y el "apeiron" sonoro. Notas para una filosofía de la música. Teorema: Revista internacional de filosofía, 31(3), 193-208.

»Mueller, C. (2012). Gothicism and English Goth Music: Notes on the Repertoire. Gothic Studies, 14(2), 74-88. https://doi.org/10.7227/gs.14.2.6

»Murray, R. (2007). The World of Jazz Trumpet: A Comprehensive History and Practical Philosophy. Music Educators Journal, 94(1), 55-56. https://doi. org/10.1177/002743210709400119

»Negretto, E. (2012). Expectation and Anticipation as Key Elements for the Constitution of Meaning in Music. Teorema: Revista internacional de filosofía, 31(3), 149-163. 
» Olteţeanu, I. (2009). Music, Rhetoric, and Representation. Analysis and Metaphysics, 08, 160-164.

»Ortiz Morales, J. M. (2010). El synchrociné o ciné-pupitre de Charles dela Commune: Un dispositivo olvidado. En M. De Aguilera, A. Sedeño, \& E. Borges Rey (Eds.), Hibridando el saber. Investigar en comunicación y música (1a ed., pp. 179-189). Málaga: Universidad de Málaga.

» Oyola Pérez, J. M., \& Borges, E. (2010). La explotación del éxito comercial a través del fenómeno de la versión. En M. De Aguilera, A. Sedeño, \& E. Borges Rey (Eds.), Hibridando el saber. Investigar en comunicación y música (1a ed., pp. 74-81). Málaga: Universidad de Málaga.

»Perman, T. (2011). The Ethics of Ndau Performance: Questioning Ethnomusicology's Aesthetics. Journal of Musicological Research, 30(3), 227252. https://doi.org/10.1080/01411896.2011.564056

»Pinilla Burgos, R. (2005). The Metaphysics of Music in Romanticism. Pensamiento, 61(231), 421-439. Recuperado de https://www.scopus.com/ inward/record.uri?eid=2-s2.0-61149423250\&partnerID=40\&md5=145242 02351afbd389e842855a40e041

»Pogăceanu, L. (2008). Musical Experience and the Perceptual Consequences of Music Performance. Analysis \& Metaphysics, 7, 226-229.

»Pogăceanu, L. (2009). Music as a manifestation of human cognitive capacities. Linguistic and Philosophical Investigations, 8, 165-169.

" Pontara, T. (2015). Interpretation, Imputation, Plausibility: Towards a Theoretical Model for Musical Hermeneutics. International Review of the Aesthetics and Sociology of Music, 46(1), 3-41.

»Puri, M. (2012). Jankélévitch and the Dilemma of Decadence. Journal of the American Musicological Society, 65(1), 241-246.

» Rings, S. (2012). Vladimir Jankélévitch's Philosophy of Music: Talking and Listening with Jankélévitch. Journal of the American Musicological Society, 65(1), 218-223.

»Rosales Meana, D. (2013). La música y los límites del mundo. Un estudio desde Eugenio Trías y Agustín de Hipona. En J. L. Villacañas Berlanga (Ed.), Anales del Seminario de Historia de la Filosofía (Vol. 30, Número 1, pp. 27-47). Madrid: Universidad Complutense de Madrid. https://doi.org/https://doi. org/10.5209/rev_ASHF.2013.v30.n1.42450

» Rowell, L. (1996). Introducción a la filosofía de la música (2a ed.). Barcelona: Gedisa.

»Sanguinetti, G. C. (2012). Música y subjetividad Hegel y las concepciones románticas de la música. En Facultad de Filosofía (Ed.), Anales del Seminario de Historia de la Filosofía (Vol. 29, Número 2, pp. 593-608). Madrid: Universidad Complutense de Madrid.

»Schiavio, A. (2012). Constituting the Musical Object: A Neurophenomenological Perspective on Musical Research. Teorema, 31(3), 
63-80. Recuperado de https://www.jstor.org/stable/43046956

"Scruton, R. (1987). Analytical Philosophy and the Meaning of Music.

The Journal of Aesthetics and Art Criticism, 46, 169-176. https://doi. org $/ 10.2307 / 431273$

»Shouse, E. (2005). Feeling, Emotion, Affect. M/C Journal, 8(6), 1-3.

»Steinberg, M. (2008). Escuchar a la razón: cultura, subjetividad y la música del siglo XIX (1a ed.). México: Fondo de Cultura Económica.

»Stratilková, M. (2016). Husserlian Notion of Inner Perception, Sound Qualities and the Philosophy of Music in Geiger. International Review of the Aesthetics and Sociology of Music, 47(2), 207-222. Recuperado de https:// www.jstor.org/stable/44234970?seq=1

"Szabados, B. (2014). Wittgenstein as Philosophical Tone-poet. Amsterdam: Rodopi. https://doi.org/10.1163/9789401210997_004

» Tarasti, E. (2008). Los signos en la historia de la música, historia de la semiótica musical. Tópicos del seminario, 19, 15-71.

» Trías, E. (2007). El canto de las sirenas. Argumentos musicales (1a ed.). Barcelona: Galaxia Gutemberg/Círculo de Lectores.

» Trías, E. (2010). La imaginación sonora (1a ed.). Barcelona: Galaxia Gutemberg.

»Van Eecke, L. (2014). Adorno's Listening to Stravinsky. Towards a Deconstruction of objectivism. International Review of the Aesthetics and Sociology of Music, 45(2), 243-260.

»Viñuela, E. (2010). El espacio urbano en la música popular: de la apropiación discursiva a la mercantilización. Trípodos. Facultat de Comunicació i Relacions Internacionals Blanquerna, 26, 15-28.

»Walser, R. (2003). Popular Music Analysis: Ten Apothegms and Four Instances. Analyzing Popular Music, 1(1), 16-38.

»Welten, R. (2009). What do We Hear When We Hear Music? A Radical Phenomenology of Music. Studia Phaenomenologica, 9, 269-286. https://doi. org/10.7761/SP.9.269

"Whale, M. (2015). How Universal is Beethoven? Music, Culture, and Democracy. Philosophy of Music Education Review, 23(1), 25. https://doi. org/10.2979/philmusieducrevi.23.1.25

»Wilson, S. (2014). After Beethoven, After Hegel: Legacies of Selfhood in Schnittke's String Quartet No. 4. International Review of the Aesthetics and Sociology of Music, 45(2), 311-334. Recuperado de https://www.jstor.org/ stable/43198650

»Woodside, J. (2008). La historicidad del paisaje sonoro y la música popular. Trans. Revista transcultural de música, 12, 2-17. Recuperado de https://www. sibetrans.com/trans/trans12/art21.htm 


\section{Biografías / Biografias / Biographies}

\section{Carlos Arango}

Comunicador, Magíster y Candidato a Doctor en Filosofía. Líder del grupo de investigación Communis, del programa Comunicación Social de la Universidad Católica de Oriente, Antioquia, Colombia. Líneas de investigación: música, imaginarios y consumo.

\section{Sergio Roncallo-Dow}

Filósofo, Magíster en Comunicación, Doctorado en Filosofía. Docente investigador de la Universidad de La Sabana, Chía, Colombia. Editor de la Revista Palabra Clave. Líneas de investigación: teoría de la comunicación, cultura popular, media ecology, exosemiótica. 